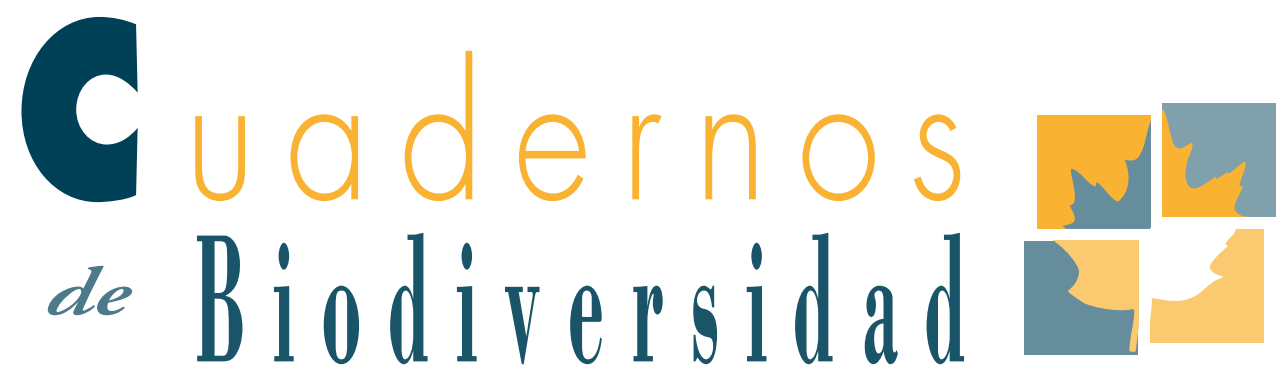

www. cuadernosdebiodiversidad.org

\title{
El efecto de la normativa de protección en la mejora de la conservación ex situ de especies amenazadas: germinación de plantas całalogadas en la comunidad vallenciana (españa)
}

\section{E. Laguna ${ }^{1}$, P. P. Ferrer Gallego ${ }^{1}$, M. C. Escribáa ${ }^{1}$ C. Peña ${ }^{2}, A$. Sebastián ${ }^{2}$ I. Ferrando ${ }^{1}$, F. J. Albert ${ }^{1}$ y A. J. Navarro ${ }^{1}$}

Generalitat Valenciana, Conselleria de Infraestructuras, Territorio y Vivienda. Servicio de Espacios Naturales y Biodiversidad.

i CiEf, Centro para la Investigación y Experimentación Forestal. Avda. Comarques del País Valencià, i i 4. 46930 Quart de Poblet, Valencia

2 CiP, Centro de Investigaciones Piscícolas El Palmar. Servicio de Espacios Naturales y Biodiversidad. C/ Francesc Cubells, i i. 460 i i Valencia.

DirecCión DE CONTACTO: LAGUNA_EMi@GVA.ES

\section{ABSTRACT}

The effect of protection rules on the improvement on the ex situ conservation of threatened species: germination of strictly protected plants in the Valencian Community

The positive effect of new legal rules improving the ex situ conservation actions on protected plants is analysed in the Valencian Community (Spain), comparing the data of seed germination tests made by the Valencian Wildlife Service (Servicio de Espacios Naturales y Biodiversidad) on the species strictly protected by a new regional Decree, entering in force in 2009. The amount of tested species after the enforcement of this Decree (years 2009-2011, $\mathrm{x}=35,000 \pm 9,644$ species/year) is significantly different $(\mathrm{F} 13,2=0,132 ; \mathrm{p}=0,013)$ than the pre-Decree period (1995-2008, $\mathrm{x}=6,500 \pm 3,503$ species/year). If only new species tested are compared -so not considering the data on formerly tested species- the difference between the pre-Decree time $(2,286 \pm$ 1,978 new species/year) and the post-Decree period $(14,333 \pm 7,767$ new species/year) is also significant (F13,2=0,065; $\mathrm{p}=0,001)$. 


\section{KEY WORDS:}

Plant Conservation laws, germination tests, protected plants, endangered species, wildlife service.

\section{INTRODUCCIÓN}

Usualmente la comunidad técnico-científica comprometida con la conservación de la flora, parte de la esperanza de que sus propuestas de protección legal de muchas especies amenazadas se reflejen en un incremento efectivo de los esfuerzos para mejorar el conocimiento, seguimiento y los trabajos para reflotar las poblaciones de tales taxones. Sin embargo, en muchas ocasiones las normas de protección de flora silvestre se han restringido a meras listas de especies protegidas, no acompañadas de directivas concretas para el desarrollo de trabajos de conservación activa. Entre las escasas normativas que articulan una completa estrategia de conservación, incluyendo mandatos precisos para el desarrollo de actuaciones y estructuras ex situ e in situ, se encuentra el Decreto 70/2009 del Consell de la Generalitat Valenciana (D.O.C.V., 2009), por el que se establece el Catálogo Valenciano de Especies de Flora Amenazada (en adelante CVEFA). El decreto protege a 398 especies, de las que 125 constituyen el CVEFA en sentido estricto, formado a su vez por 42 taxones en la categoría 'En Peligro de Extinción' (en adelante EP) y 83 como 'Vulnerable' (VU). Los máximos niveles previstos en el ordenamiento jurídico español y la descripción detallada de los 125 taxones catalogados en la Comunidad Valenciana está accesible en el trabajo de AGUILELLA et al. (2010).

La norma incluye un amplio elenco de medidas de impulso de la conservación activa y del fomento de la participación pública, desde el reconocimiento oficial de herbarios y colecciones botánicas, hasta la creación del Banco de Germoplasma de Flora Silvestre Valenciana (BGFSV, con sede en el Jardín Botánico de la Universidad de Valencia), pasando por la creación del recolector científico y por el reconocimiento de personas y entidades colaboradoras. La norma establece un mandato concreto a las unidades técnicas de la administración pública res- ponsable de su ejecución (la Generalitat Valenciana, gobierno regional de la Comunidad Valenciana), que se centran especialmente en materias del Servicio de Espacios Naturales y Biodiversidad (SENB). Dicho servicio se encargó de las fases técnicas preliminares de elaboración del decreto (AGUILELLA et al., op. cit.), por lo que con antelación a su aprobación, a finales de 2008, replanteó su funcionamiento para adaptarse al nuevo escenario normativo y funcional que generaría dicha norma (LAGUNA, 2008). Con antelación al trámite del CVEFA, la gestión de la flora singular valenciana se había basado en dar prioridad a las especies endémicas con suficiente grado de rareza, estuviesen o no amenazadas (LAGUNA, 2002; ESCRIBÁ et al., 2002), siendo su resultado más tangible la red de microrreservas (LAGUNA, 2008). En el nuevo enfoque, adoptado ya desde el inicio de 2009, se pasa a priorizar las especies amenazadas, fuesen o no endémicas, un matiz que entre otras cosas refuerza el interés por la conservación de los tipos relictos de vegetación, habitualmente pobres en endemismos pero ricos en especies raras amenazadas, o el de figuras normativas hasta entonces poco potenciadas como los planes de recuperación y conservación.

Como consecuencia de este cambio de estrategia, dos unidades técnicas del SENB dedicadas parcialmente a la conservación ex situ, recibieron la orden de reorientar sus criterios de actividad: de un lado el Centro de Investigaciones Piscícolas (CIP, El Palmar, Valencia), donde se desarrollan los trabajos de conservación ex situ de flora acuática y palustre valencianas (SEBASTIÁN et al., 2008) y de otro, el equipo de trabajo e instalaciones del citado servicio en el Centro para la Investigación y Experimentación Forestal (CIEF, Quart de Poblet, Valencia). Este centro realiza funciones similares para la flora terrestre (ESCRIBÁ et al., 2002, 2006); en ambos casos los primeros trabajos de puesta en germinación y cultivo de plantas amenazadas se iniciaron en 1995, y su desarrollo sigue procesos y protocolos similares a los indicados por BACCHETTA et al. (2008). Dado que el cambio de orientación temática en las directrices de conservación se hizo efectivo con la transición de año de 2008 a 2009 y resulta factible comparar el efecto de su entrada en vigor, 
comparando los datos de actuaciones concretas con antelación (de 1995 a 2008) y posterioridad, para el periodo 2008-2011. En el presente trabajo se analiza el efecto de este cambio de estrategia en lo referente a la conservación ex situ, en particular para el desarrollo de trabajos de puesta en germinación de las especies amenazadas.

\section{MATERIAL Y MÉTODOS}

Se han analizado las series de datos de actividad en germinación de las 125 especies amenazadas del CVEFA realizados en el CIP y el CIEF desde 1995 a 2011, que se encuentran disponibles mediante tabla sintética en la dirección web http:// www.uv.es/elalum/germ/GerminacionesCVEFA_ SENB1995a2011.pdf Para muchas de estas especies existen además fichas detalladas de información y protocolos de germinación en el reciente trabajo de FERRER et al. (2012).

A efectos de su estudio y tratamiento estadístico, los datos se han subdividido en dos tramos: etapa anterior a la entrada en vigor del Catálogo Valenciano de Especies Amenazadas(tramo pre-CVEFA, 1995-2008) y posterior a dicho momento (CVEFA, 2009-2011). Para el análisis se han utilizado dos tipos de información: 1) Matriz de datos sobre las especies en las que se han realizado trabajos de germinación de cualquier tipo cada ańo, y 2) igual que el anterior, pero con referencia a las especies que se han germinado por primera vez en las unidades del SENB. La diferencia entre ambas series es que en la primera, una especie puede aparecer en varios ańos, p.ej. plantas que se someten a tests secuenciados interanuales de germinación para conocer el efecto de sus técnicas de almacenamiento en los bancos de germoplasma, pérdida de viabilidad, etc., mientras en el segundo cada taxón sólo computa para un año concreto, aquel en el que por primera vez se realizaron pruebas germinativas.

Se han considerado los datos de todo tipo de experiencias de germinación, desde pruebas iniciales hasta aquellas que han generado protocolos de germinación estandarizados conforme a las normas regularmente actualizadas por la International Seed Testing Association (I.S.T.A., 2012), con o sin pretratamientos concretos, o las de tratamientos en sentido estricto. Los datos de la comparación preCVEFA vs CVEFA se han realizado mediante análisis simple de la varianza, considerando los años como repeticiones, y siendo las unidades de medición el número de especies germinadas por año, ya fueran totales en el caso 1) o nuevas sin pruebas precedentes en el caso 2). La hipótesis nula (Ho) implicaba la ausencia de diferencias significativas entre los dos bloques de datos, y en consecuencia la falta de efecto de la orden de reorientación de actividad impulsada por el Decreto 70/2009, mientras la alternativa (H1) implica que se encuentran diferencias con mayor número de especies catalogadas con pruebas de germinación para el período 2009-2011.

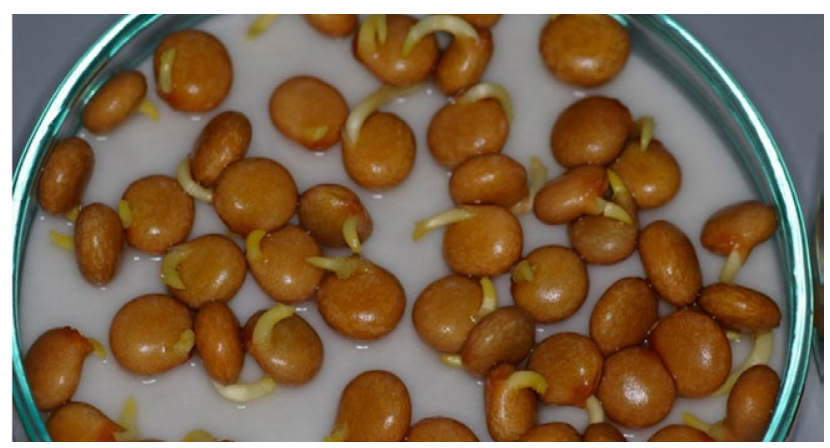

Germinación de Lupinus mariae-josephae H. Pascual (Leguminosae), especie protegida en la categoría Vulnerable por el Decreto 70/2009

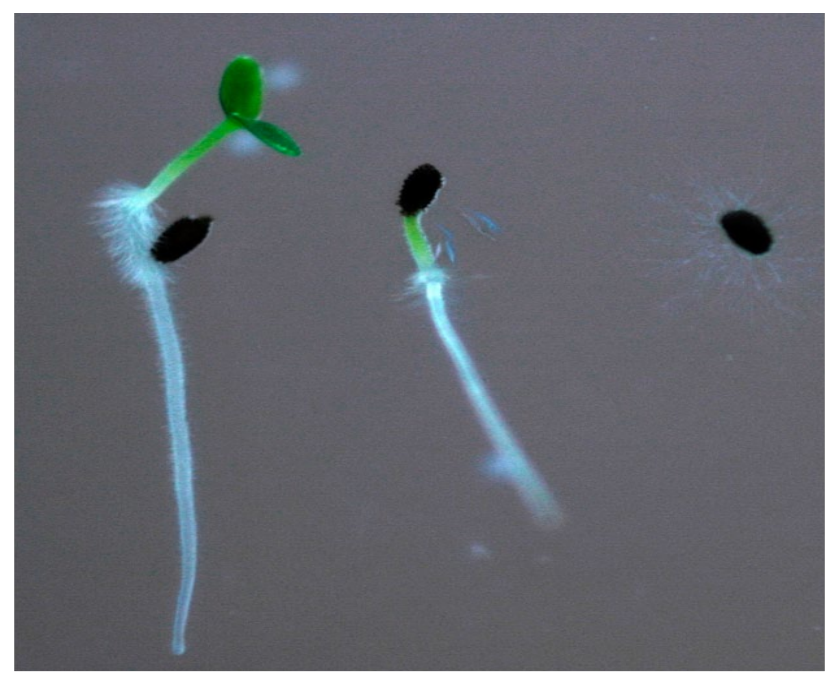

Germinación de la especie Garidella nigellastrum L. (Ranunculaceae) especie que figura en la categoría En Peligro de Extinción en el Decreto 70/2009 


\section{RESULTADOS Y DISCUSIÓN}

Considerando la globalidad del período de muestreo, 1995-2011, las instalaciones del SENB han albergado pruebas de germinación en 29 especies EP $(69,05 \%$ del total protegido en esa categoría) y 46 VU (55,42\%), según se expone en la Fig. 1; en general las especies para las que no se han desarrollado trabajos (50 taxones, correspondientes al $40 \%$ del total del CVEFA) son plantas para las que se carece de tecnología óptima, p.ej. orquídeas, briófitos como Riella helycophylla, etc., pteridófitos que ya se han germinado en líneas específicas que desarrolla el Jardín Botánico de la Universidad de Valencia, especies extintas, plantas que ya poseen protocolos comerciales testados y son por tanto menos urgentes para someter a estudios de detalle (p.ej., Ribes uva-crispa, Ruscus hypophyllus, etc.), árboles y grandes arbustos cuya germinación se aborda por otras unidades de la Generalitat Valenciana, en concreto el Banco de Semillas Forestales del propio CIEF, como ocurre con Tilia platyphyllos, Ulmus glabra, Cotoneaster granatensis, etc., o bien aquellas especies que no parecen producir hasta ahora semillas fértiles (p.ej. Narcissus perezlarae) y que tienden a propagarse alternativamente por vía vegetativa. El estudio abordado ha detectado no obstante algunos huecos significativos, como la falta de pruebas de germinación para una especie valenciana de la Directiva de Hábitats, Euphorbia nevadensis subsp. nevadensis, que debería abordarse a la mayor brevedad, dada la especial rareza territorial de este taxón (v. AGULELLA et al., 2010).
El número de especies para las que se han desarrollado trabajos de germinación cada año se expresa en la Fig. 2 y la Tabla 1, donde puede apreciarse directamente que el resultado del esfuerzo abordado en el período CVEFA (2009-2011) redunda en una diferencia significativa en el número de especies objeto de experiencias. Considerando todos los taxones catalogados, se ha pasado de un promedio de 6,5 especies año a 35; como puede apreciarse en la Tabla 1, estas proporciones serían aún mayores si hablamos de los taxones En Peligro de extinción, donde casi se multiplica por 8 el número de especies estudiadas.

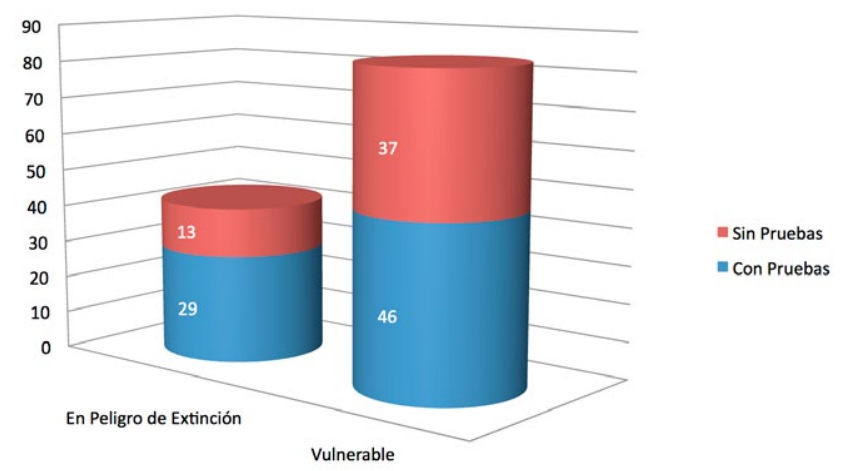

Figura 1. Representación del número de especies para las que se han desarrollado (en azul) y aún falta (en rojo) pruebas de germinación en instalaciones del SENB

\begin{tabular}{|c|c|c|c|c|c|}
\hline & Período & $n^{0}$ especies/año & $F_{13,2}$ & p (bilateral) & $\begin{array}{l}\text { Hipótesis } \\
\text { válida }\end{array}$ \\
\hline \multirow{2}{*}{$\begin{array}{l}\text { En Peligro de } \\
\text { Extinción }\end{array}$} & PreCVEFA & $1,857 \pm 1,231$ & \multirow{2}{*}{0,123} & \multirow{2}{*}{0,010} & \multirow{2}{*}{$\mathrm{H}_{1}$} \\
\hline & CVEFA & $14,667 \pm 3,512$ & & & \\
\hline \multirow{2}{*}{ Vulnerable } & PreCVEFA & $4,643 \pm 2,706$ & \multirow{2}{*}{0,182} & \multirow{2}{*}{0,037} & \multirow{2}{*}{$\mathrm{H}_{1}$} \\
\hline & CVEFA & $20,333 \pm 6,351$ & & & \\
\hline \multirow{2}{*}{ Total Catalogadas } & PreCVEFA & $6,500 \pm 3,503$ & \multirow{2}{*}{0,132} & \multirow{2}{*}{0,013} & \multirow{2}{*}{$\mathrm{H}_{1}$} \\
\hline & CVEFA & $35,000 \pm 9,644$ & & & \\
\hline
\end{tabular}

Tabla 1. Resultados del análisis de la varianza para la comparación del número de especies sometidas a pruebas de germinación por cada año. 


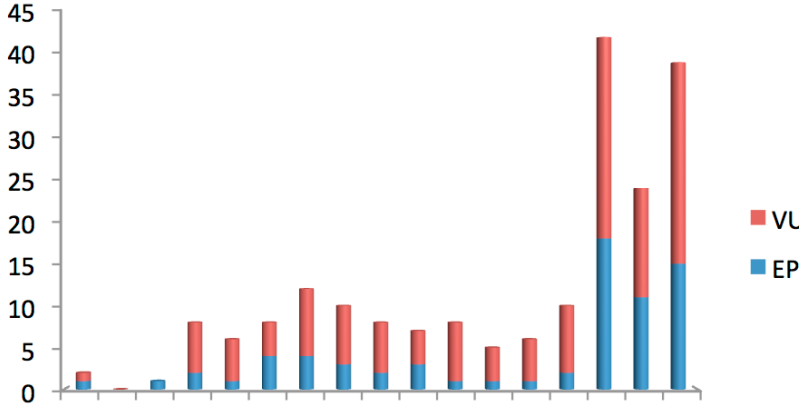

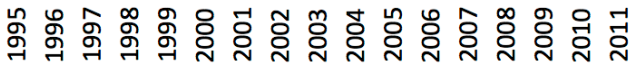

Figura 2. Número de especies catalogadas (EP: En Peligro de Extinción, VU: Vulnerable) objeto de trabajos de germinación en el período 1995-2011. Eje x: Años, Eje Y: $n^{\circ}$ especies.

Cuando consideramos exclusivamente los datos para especies germinadas por primera vez en el CIP y/o CIEF, los resultados obtenidos son los expresados en la Fig. 3 y la Tabla 2, que siguen la tónica de los indicados anteriormente, aunque con la salvedad de que las diferencias no son significativas para estimación bilateral de $\mathrm{p}$ en el caso de los taxones Vulnerables.

Las razones de este menor grado de diferenciación en la categoría Vulnerable se hallan a nuestro entender en dos causas: 1) el hecho de que los taxones con los que ya se ha realizado alguna vez una prueba de germinación no computan ya en este segundo tipo de series de datos, por lo que lo esperable es un descenso progresivo del número de

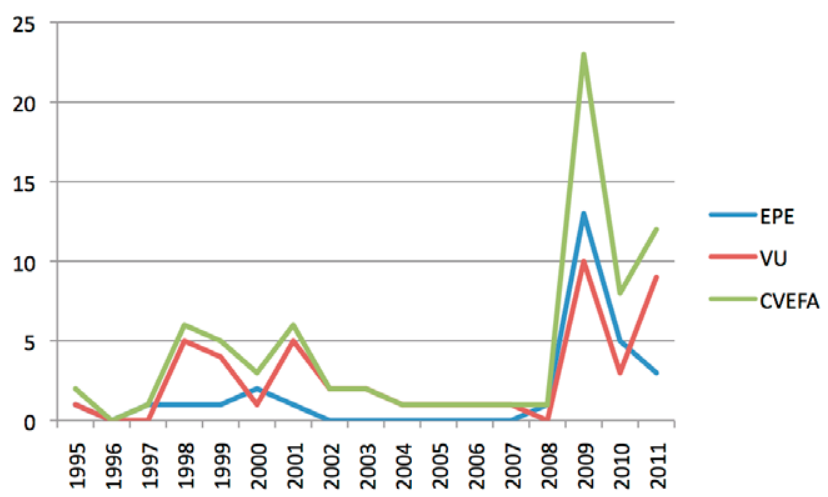

Figura 3. Número de especies catalogadas con primeras experiencias de germinación en el período 1995-2011

especies germinadas por año, y 2) que en algunos de los años del período pre-CVEFA (en concreto 1998 y 2001) se realizó un esfuerzo especial en la puesta en germinación de especies de la Directiva de Hábitats, en su mayoría catalogadas como Vulnerable en el CVEFA, de las que se había podido obtener germoplasma de adecuada calidad, ya que dichos trabajos formaban parte de los compromisos de ejecución de los proyectos LIFE desarrollados por el SENB y detallados por LAGUNA (2002) y LAGUNA et al. (2003). Las causas indicadas fuerzan la existencia de valores elevados en las desviaciones típicas de la Tabla 2, que a su vez condicionan un menor nivel de significación en la prueba de Fisher para la categoría citada. De hecho, y aunque las proporciones CVEFA/PreCVEFA para los datos medios $(\mathrm{x})$ son

\begin{tabular}{|c|c|c|c|c|c|}
\hline Categoría & Período & $n^{0}$ especies/año & $\mathrm{F}_{13,2}$ & p (bilateral) & $\begin{array}{l}\text { Hipótesis } \\
\text { válida }\end{array}$ \\
\hline \multirow{2}{*}{$\begin{array}{l}\text { En Peligro de } \\
\text { Extinción }\end{array}$} & PreCVEFA & $0,538 \pm 0,660$ & \multirow{2}{*}{0,016} & \multirow{2}{*}{$<0,0001$} & \multirow{2}{*}{$\mathrm{H}_{1}$} \\
\hline & CVEFA & $7,000 \pm 5,292$ & & & \\
\hline \multirow{2}{*}{ Vulnerable } & PreCVEFA & $1,714 \pm 1,729$ & \multirow{2}{*}{0,209} & \multirow{2}{*}{0,055} & \multirow{2}{*}{$\mathrm{H}_{\mathrm{o}}$} \\
\hline & CVEFA & $7,333 \pm 3,786$ & & & \\
\hline \multirow{2}{*}{ Total Catalogadas } & PreCVEFA & $2,286 \pm 1,978$ & \multirow{2}{*}{0,065} & \multirow{2}{*}{0,001} & \multirow{2}{*}{$\mathrm{H}$} \\
\hline & CVEFA & $14,333 \pm 7,767$ & & & \\
\hline
\end{tabular}

Tabla 2. Resultados del análisis de la varianza para la comparación del número de especies sometidas a pruebas de germinación por cada año. 
aparentemente elevadas para las especies Vulnerables $(4,28=428 \%)$, si se considera el número total de especies germinadas por primera vez para cada serie completa (13 años Pre-CVEFA y 3 CVEFA, ver Fig. 4), hasta el momento la suma de taxones es aún superior en el primer período, aunque obviamente se prevea cambiar este balance cuando se acumulen los nuevos datos de ańos sucesivos.

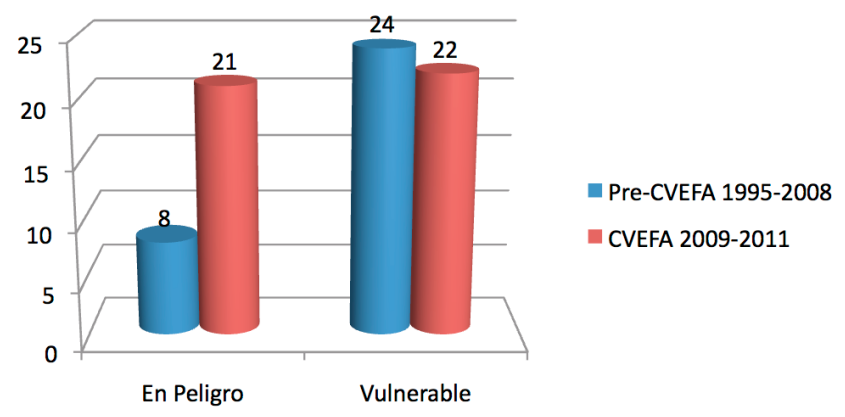

Figura 4. Representación del número de especies para las que se han hecho pruebas de germinación en cada uno de los 2 períodos de estudio (Pre-CVEFA 1995-2008 y CVEFA 2009-2011), en cada una de las dos categorías de especies catalogadas.

Como conclusión, los resultados indican una clara influencia de la aprobación del Catálogo Valenciano de Especies de Flora Amenazadas en el desarrollo de trabajos de conservación con las especies que contiene, aunque es importante reseñar que dicho avance debe considerarse como efecto combinado de un posicionamiento del servicio gestor (SENB), transferido en forma de directrices concretas, y del contenido de la norma, que a diferencia de otras parecidas en la mayoría de regiones españolas y europeas, ha incluido una amplia estrategia de actividad de conservación y el subsecuente mandato para su ejecución. En consecuencia conviene recomendar que las futuras normas similares en otros territorios incluyan, como en el caso de la valenciana, directrices susceptibles de conformar una estrategia completa de conservación, en vez de restringirse a simples listas de especies protegidas.

\section{AGRADECIMIENTOS}

Las actividades aquí indicadas han sido cofinanciadas por la Comisión Europea a través de diversos proyectos LIFE y del programa operativo de los fondos FEOGA-Orientación y FEADER en la Comunidad Valenciana.

\section{REFERENCIAS}

AGUILELLA, A., E. LAGUNA, E. \& S. FOS. 2010. Catálogo Valenciano de Especies de Flora Amenazadas. Generalitat Valenciana, Valencia.

http://www.cma.gva.es/comunes_asp/documentos/ agenda/Cas/65557-CatalogoFloraAmenazada.pdf

BACCHETTA, G., A. BUENO, G. FENU, B. JIMÉNEZALFARO, E. MATTANA, B. PIOTTO \& M. VIREVAIRE 2008. Conservación ex situ de flora silvestre. Obra Social La Caixa y Gobierno del Principado de Asturias. Oviedo. 378 pp.

http://www.asturias.es/medioambiente/publicaciones/ ficheros/Conserv-exsitu.pdf

D.O.C.V.. 2009. Decreto 70/2009, de 22 de mayo, del Consell, por el que se crea y regula el Catálogo Valenciano de Especies de Flora Amenazadas y se regulan medidas adicionales de conservación. Diari Oficial de la Generalitat Valenciana , 6021 (26.05.2009): 20.143-20.162

http://web.uam.es/otros/consveg/documentos/valencia.pdf

ESCRIBÁ, M.C., E. LAGUNA \& M. GUARA. 2006. Seed germination trends of endemic vascular plants in the Valencian Community (Spain). En: Aguilella, A., A. M. Ibars, E. Laguna \& B. Pérez Rocher (eds.): Proceedings of the 4 th European Conference on the Conservation of Wild Plants. 4pp, $63 \mathrm{~Kb}$, ext PDF. Generalitat Valenciana y Universitat de València. Valencia.

http://www.nerium.net/plantaeuropa/Download/Procedings/Escriba_et_al.pdf

ESCRIBÁ, M.C., A. OLIVARES, G. BALLESTER, V.I. DELTORO, C. FABREGAT, S. FOS, E. LAGUNA, J. PÉREZ-BOTELLA, P. PÉREZ-ROVIRA \& L. SERRA. 2002. Germinación de taxones de flora rara, endémica o amenazada en la Comunidad Valenciana. En: EspiritoSanto, D., J.C. Costa \& A.L. Soares (eds.): Jardins Botânicos. Que perspectiva para o futuro?, pp. 197-202. Associaçao Íbero-Macaronésica de Jardins Botânicos e Instituto Superior de Agronomia da Universidade de Lisboa. Lisboa. 
FERRER, P.P., I. FERRANDO, C. GAGO \& E. LAGUNA. 2012. Manual para la conservación de germoplasma y el cultivo de la flora valenciana amenazada. Col. Manuales Técnicos de Biodiversidad, no 3 (edición en CD). Conselleria de Infraestructuras, Territorio y Medio Ambiente. Generalitat Valenciana. Valencia. 250 pp.

I.S.T.A. 2012. International rules for seed testing. Edition 2012. International Seed Testing Association. Bassersdorf. 425 pp.

LAGUNA, E. 2002. Estrategia de conservación de la flora silvestre en la Comunidad Valenciana. Conservación Vegetal 7: 12-13

http://web.uam.es/otros/consveg/documentos/numero7.pdf
LAGUNA, E. 2008. La conservación de la Biodiversidad aplicada a pequeña escala: La red valenciana de microrreservas de flora. En: Grisolía, S. (coord.): Biodiversidad, pp. 249-263. Presidència de la Generalitat Valenciana Fundación Premios Jaime I. Valencia.

http://www.uv.es/elalum/artmicros/2008_Laguna_Biodiversidad.pdf

LAGUNA, E., G. BALLESTER, A. OLIVARES, L. SERRA, P. PÉREZ ROVIRA, V.I. DELTORO, J. PÉREZ BOTELLA \& S. FOS. 2003. Conservation of priority habitats in the Valencian Community, Spain (Project LIFE99 NAT/E/006417). Ecologia Mediterranea 29(1): 109.

SEBASTIÁN, A., C. PEÑA \& E. LAGUNA. 2008. Experiencias de conservación de balsas temporales y otras zonas húmedas en el territorio valenciano. En: Vila, X., M. Campos \& C. Feo (eds.): Conservació, problemàtiques i gestió de les llacunes temporànies mediterrànies, pp. $207-$ 223. CD-ROM. Consorci de l'Estany. Girona. 